\title{
Identification of Aberrantly Expressed Genes in Murine Glioblastoma During Radiotherapy via Bioinformatic Data Mining
}

This article was published in the following Dove Press journal: OncoTargets and Therapy

\author{
Xihe Zhao' \\ Chenxi $\mathrm{Li}^{2}$ \\ Lei Liu $^{3}$ \\ Huawei Zou' \\ Kai Li ${ }^{1}$ \\ 'Department of Clinical Oncology, \\ Shengjing Hospital of China Medical \\ University, Shenyang I I0004, People's \\ Republic of China; ${ }^{2}$ Department of \\ Radiation Oncology, Shenyang Cancer \\ Hospital, Shenyang II 0023, People's \\ Republic of China; ${ }^{3}$ Department of \\ General Surgery, Shengjing Hospital of \\ China Medical University, Shenyang \\ I 10004, People's Republic of China
}

Correspondence: Kai $\mathrm{Li}$

Department of Clinical Oncology,

Shenging Hospital of China Medical

University, Shenyang II0004, People's

Republic of China

Email lik@sj-hospital.org
Objective: Glioblastoma (GBM) is an aggressive tumor with a fast growth rate. Radioresistance of GBM can lead to high recurrence. In general, due to the protection of the blood-brain barrier, the immune environment of the central nervous system is unique. The immune response induced by radiotherapy is weak in GBM. In the present study, aberrantly expressed genes during radiotherapy were assessed in murine models based on microarray RNA data.

Methods: The microarray data were extracted from the Intergovernmental Group on Earth Observations and differentially expressed genes (DEGs) screened out. Gene expression profiles of 115 samples in GSE56113 were analyzed and 104 genes were identified as aberrantly expressed based on GEO2R $8 \mathrm{~d}$ after radiotherapy. Then, the Database for Annotation, Visualization, and Integrated Discovery was used to analyze Genome Kyoto Encyclopedia of Gene pathways and Gene Ontology (GO) terms. The 20 core candidate genes were identified using protein-protein interaction network analysis and Cytoscape software with Molecular Complex Detection plug-in.

Results: Post-irradiated tumor tissues expressed significantly more immune-associated genes than contralateral brain tissues. GO and pathway analyses showed core DEGs were mainly enriched in the chemokine signaling and IL-6 signaling pathways, which could lead to immunosuppressive inflammatory monocyte infiltration and radioresistance. Chemokine signaling and IL-6 signaling pathway-associated genes were increased in the irradiated U87 cell strain.

Conclusion: Chemokine signaling and IL-6 signaling pathways were activated after radiation in murine glioma and human glioma cell lines which could lead to changes in the immune microenvironment and treatment failure. The results of the present study could provide potential therapeutic targets especially when immune therapy and radiotherapy are combined to treat GBM patients.

Keywords: glioblastoma, immune microenvironment, radiotherapy, bioinformatic data mining

\section{Introduction}

Glioblastoma (GBM) is one of the most common intracranial malignant tumors. ${ }^{1}$ Currently, the main treatment for GBM is surgical resection plus adjuvant radiotherapy and chemotherapy. ${ }^{2}$ Radiotherapy is a benchmark therapy for local control of tumors; however, GBM rarely shows a strong response to radiotherapy. ${ }^{3}$ Tumor radiotherapy is mainly based on the penetration of radiation and the biological ionizing of cells. Biologically active human tissues absorb ionizing radiation energy and produce a series of extremely complex physical, chemical, and biological 
changes, which eventually lead to biological damage to tissues. ${ }^{4}$ In recent years, researchers have focused on the spontaneous regression of distant metastases and the damage to normal tissues in non-irradiated fields after local radiotherapy (abscopal effect). ${ }^{5}$ Radiotherapy may become a method of systemic immune system activation and play an important role in the development and prognosis of malignant tumors. Tumor antigens released by necrotic cells can stimulate immune responses in vivo. A radiation-targeted tumor and its microenvironment initiate inflammatory response loops and manifest systemic immunologic consequences. ${ }^{6}$ The immune response generated by radiotherapy can be a benefit that is also a liability; ${ }^{7}$ radiotherapy can stimulate anti-tumor immune response as well as immunosuppressive response. ${ }^{8}$

Generally, intracranial immunologic response is absent during the radiotherapy process. The concept that the central nervous system (CNS) is immune-privileged was based on the intracranial xenograft experiment in rodents ${ }^{9}$ because the blood-brain barrier (BBB) could protect the brain. In addition, dedicated lymphatic channels were believed lacking in the CNS. Supposedly, the antigen presentation of intracranial tumor antigens to immune cells was limited and immune cells could barely penetrate the BBB. ${ }^{10}$

Microglial cells were considered major antigenpresenting cells for brain tumors and showed suppressive function for cytotoxic $\mathrm{T}$ cells. ${ }^{11,12}$ A novel route of lymphatic egress from the brain along distinct channels was investigated in 2015. Most antigen-presenting cells may travel parallel to dural venous sinuses to the deep cervical lymph nodes, then prime $\mathrm{T}$ and $\mathrm{B}$ lymphocytes. ${ }^{13}$ In the present study, a series of different immunotherapies for GBM were performed due to advances in immunotherapy in other tumors. Post-radiotherapy immune response of GBM was investigated. Thus, identification of radiotherapy-associated gene changes and investigation of their clinical roles and molecular mechanisms are essential for understanding the cause of treatment failure.

Genomic testing has provided powerful detection tools for new therapeutic targets. High-throughput gene sequencing technologies are widely used to comprehensively analyze differentially expressed genes (DEGs). The intergovernmental Gene Expression Omnibus (GEO) has facilitated the analysis of high-throughput data of various genomic alterations. ${ }^{14,15}$ However, due to ethical limitations, human post-radiotherapy GBM samples can rarely be obtained. Murine GBM models were constructed to simulate and substitute the human in vivo environment. In the present study, the original microarray dataset GSE56113 (unpublished data) was downloaded from NCBI-GEO (https:// www.ncbi.nlm.nih.gov/geo) and the aberrantly expressed genes were identified for post-radiotherapy murine GBM. Subsequently, Gene Ontology (GO) terms and pathway enrichment analysis were developed for screening DEGs with DAVID and Panther (http://www.pantherdb.org). The DEGs protein-protein interaction (PPI) network was developed by STRING (https://string-db.org/) and core genes identified using Cytoscape software with Molecular Complex Detection (MCODE) plug-in. The distinct gene expression patterns between non-treated and irradiated GBM cells, and GBM cells and contralateral brain cells, were investigated.

The aim of the study is to investigate the rat GBM gene expression changes after radiotherapy. To find some clue on reversing glioma radioresistance and the possibility of combination of radiotherapy and immune therapy. Identifying DEGs and determining their biological functions and key pathways will provide more specific and reliable biomarkers to explain radiotherapy failure and help reverse radioresistance.

\section{Materials and Methods Microarray Data Information}

The gene expression profiles of GSE56113 were obtained from the GEO database, a free database of microarray/gene profiles and next-generation sequencing. GSE56113 was based on GPL1355 platforms (Affymetrix GeneChip ${ }^{\circledR}$ Rat 230_2) and included 115 murine tissues (13 untreated tumor tissues, 45 irradiated tumor tissues, 13 untreated contralateral tissues, and 43 irradiated contralateral tissues). This dataset was chosen to perform an integrated analysis. The raw data of highthroughput functional genomic expression were integrated and the TXT format data processed using GEO2R online tools. Gene expression profiles were initially obtained at different time points $(6 \mathrm{~h}, 48 \mathrm{~h}$, and $8 \mathrm{~d}$ ) after irradiation with one of the two radiotherapy methods, Synchrotron microbeam radiation therapy (MRT) or broad-beam (BB) and compared with untreated GBM cells using GEO2R online tools.

\section{Exploration of the Aberrantly Expressed Genes}

After the microarray RNA data of radiotherapy that included 60,483 mRNAs, as described by NCBI (https:// www.ncbi.nlm.nih.gov/) were downloaded, DEGs were 
identified using the classical $t$-test. Statistically significant DEGs were defined with adjusted $\mathrm{P}<0.05$ (Benjamini \& Hochberg correction) and the absolute $\log 2$ fold change (FC) $>2$ as the cut-off criterion. Then, the raw data in TXT format were analyzed in Venn software online to detect the common DEGs among the two different radiotherapy methods.

\section{GO and Pathway Enrichment Analyses}

Common candidate DEGs obtained at $8 \mathrm{~d}$ after MRT and $\mathrm{BB}$ were used for GO and pathway enrichment analyses. Candidate DEG functions and pathway enrichment were analyzed using the DAVID website (https://david.ncifcrf. gov/) and Panther (http://www.pantherdb.org). DEGs were classified based on the GO database annotation and KEGG Database for Annotation and Visualization.

\section{Integration of the PPI Network}

For further functional investigation, candidate DEGs PPI information was developed using the STRING online tool (https://string-db.org). High-confidence interaction genes were chosen by calculating the combined scores using the confidence score $>0.4$ as the cut-off value. Cytoscape software (http://cytoscape.org/) was applied to visualize the potential correlation between the DEGs in the irradiated tumors. Furthermore, the MCODE, a Cytoscape plug-in, was used to select modules of the PPI network (degree cutoff $=2$, max. depth $=100, \mathrm{k}$-core $=2$, and node score cutoff $=0.2$ ).

\section{Survival Analysis Based on the Kaplan-Meier Plotter Online Tool}

Core genes obtained from STRING and Cytoscape software were used for prognosis analysis conducted using UALCAN (http://ualcan.path.uab.edu/).

\section{Validation Based on Radioresistant Cell Strain}

To verify the DEGs and pathway change in the human cell line, radioresistant human GBM cell strain (U87) was established. Human glioma cell lines (U87) were purchased from Shanghai Institutes for Biological Sciences Cell Resource Center, and attachment cultured in Dulbecco's modified Eagle medium (DMEM)(Life Technologies, Invitrogen ${ }^{\mathrm{TM}}$, Cat No. 11995-073)/high glucose with 10\% FBS (Life Technologies Corporation, Carlsbad, CA, USA, Cat no. 26140-079). All cells were maintained in a humidified incubator at $37{ }^{\circ} \mathrm{C}$ with $5 \%$ $\mathrm{CO}_{2}$ (Smart Cell HF-90, Likang Inc., Shanghai, China).

For human U87 glioma cells, the medium was changed every $48 \mathrm{~h}$, cells went trypsinizing digestion and subculturing when cells were at $90 \%$ confluence rate (nearly every 4-5 days).

For U87 glioma cells, $90 \%$ confluence rate at $75 \mathrm{~cm} 2$ cell culture flasks was about $10^{\wedge} 6$ cells. U87 cells $\left(10^{\wedge} 6\right)$ in the logarithmic phase were selected and seeded in $75 \mathrm{~cm}^{2}$ cell culture flasks. Radiation experiments were performed 48 $\mathrm{h}$ later with gradient dose irradiation. Cells were exposed 3 times to 1 Gy irradiation, 3 times to 2 Gy irradiation, and 7 times to $4 \mathrm{~Gy}$ irradiation. After each irradiation, the cells were restored to the logarithmic phase for the next irradiation. When irradiated, 1.5-cm-thick tissue-equivalent fillers were placed on the top and bottom of the cell flask. The morphological changes of the cells were observed under a microscope until the cells were disintegrated and stabilized to establish the radiotolerant GBM cell line, U87R, to investigate the changes in chemotaxis and IL-6 pathways in GBM cells before and after radiotherapy. The levels of Ccl-2, Ccl-5, Ccl-7, TLR1, IL-1B, and IL-6 were detected in U87R cells and U87 cells (the parent strain).

\section{RNA Extraction and Real-Time PCR}

Total RNA was isolated from cells with Trizol reagent (Life Technologies Corporation, Carlsbad, CA, USA, Cat No. 15596-026). RNA concentration and quality were determined via 260/280 nm absorbance with Nanodrop Spectrophotometer (ND-100, Thermo, USA) (at 260/280 $\mathrm{nm}$ absorbance). One-Step SYBR Prime-Script RT-PCR Kit (TakaraBio, Inc., Japan, Code RR096) was used to detect the expression of target genes using 7500 Fast RTPCR System. GAPDH was used as the endogenous control. The expression levels were normalized to those of the endogenous controls, and fold changes were calculated using the relative quantification $\left(2^{-\Delta \Delta \mathrm{Ct}}\right)$ method.

\section{Results \\ Identification of DEGs}

GEO is a free database of microarray gene profiles and RNA sequencing and was used to obtain the gene expression profiles of untreated tissues and irradiated tissues in GSE56113. Gene expression profiles were initially obtained at different time points ( $6 \mathrm{~h}, 48 \mathrm{~h}$, and $8 \mathrm{~d})$ after radiation with one of the two radiotherapy methods, MRT and BB, and compared with untreated GBM cells using GEO2R online tools. 
For different radiotherapy methods, we analyzed the differentially expressed genes, and we take the intersection part of two methods (Venn diagram) to reduce the impact of different radiation methods and to find the common changes. The expression level of each gene transformed with $\log 2$ was calculated using DESeq R. Between the two radiotherapy methods, 22, 8, and 395 upregulated DEGs, and 3, 8, and 179 downregulated DEGs were identified at $6 \mathrm{~h}, 48 \mathrm{~h}$, and 8 d post-radiation, respectively. Next, the Venn diagram software was used to identify the common DEGs between MRT and BB methods (Figure 1). Results showed 7, 2, and 104 upregulated genes $(\log 2 \mathrm{FC}>2)$ (Figure $1 \mathrm{~A}-\mathrm{C})$ and 1,0 , and 13 downregulated genes $(\log 2 \mathrm{FC}<2)$ were detected (Figure 1D-F). The up-regulated and down-regulated DEGs gene names were represented in the supplementary material. The gene expression at $8 \mathrm{~d}$ showed the most number of DEGs and was considered the most stable gene expression change; thus, in the subsequent analysis, $8 \mathrm{~d}$ was chosen as the main time point for analysis (Figure 1C, F).

We chose an $8 \mathrm{~d}$ point because it was stable. In our past experiment, for one time high dose radiation, 8-10 days after radiation the cells on flask could grow into cell spheres and it represented the radioresistance existed. In our study, we chose 1 Gy irradiation 3 times, 2 Gy irradiation for 3 times and 4 Gy irradiation for 7 times, because the cells could possess strong resistance gradually and became stable radioresistant cells. Actually, the cells showed the trend of resistance and viability in about $8-10$ days.

Gene expressions after MRT and BB at the $8 \mathrm{~d}$ time point are represented as a heatmap (Figure 2A, B). Comparisons of DEGs after MRT versus BB, MRT versus no radiation, and $\mathrm{BB}$ versus no radiation are represented as volcano plots (Figure 2C). The DEG trends were similar between MRT and BB methods.

\section{Function and Pathway Enrichment Analyses}

Both GO and KEGG searches of the common DEGs identified between MRT and BB at the $8 \mathrm{~d}$ time point were conducted using the online tool DAVID and Panther software with $\mathrm{P}<$ 0.05 as the cut-off criterion. The GO analysis results are shown in Table 1 and KEGG results in Table 2. The DEGs were sorted into three functional groups: biological process, cellular component, and molecular function. The biological process group is shown in Table 1; upregulated genes were mainly enriched in inflammatory response, chemokine-mediated signaling pathway, immune response, and $\mathrm{T}$ cell differentiation. In the cellular component group, upregulated genes were mainly enriched in the external side of the plasma membrane,
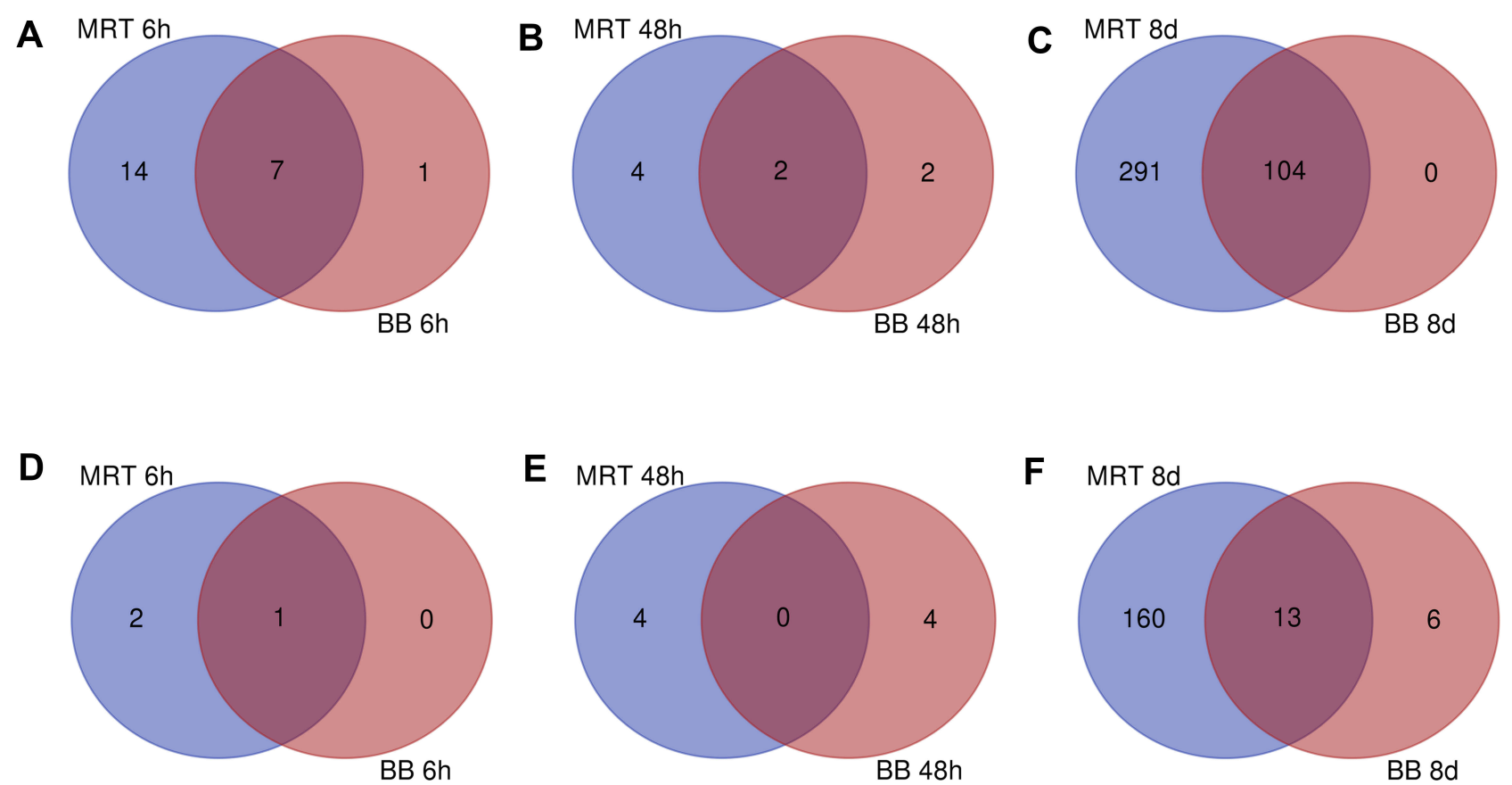

Figure I Identification of common DEGs (differentially expressed genes) in the datasets (GSE56II3) through Venn diagram software. Rats untreated vs radiated GBM (glioblastoma) samples of two radiotherapy methods (Synchrotron microbeam radiation therapy-treated tumors, MRT; broad-beam-treated tumors, BB) at different time points $(6 \mathrm{~h}, 48 \mathrm{~h}, 8 \mathrm{~d})$ were presented. Different color areas represented different radiotherapy method. The cross areas meant the commonly changed DEGs. (A-C). Upregulated DEGs at $6 \mathrm{~h}, 48 \mathrm{~h}, 8 \mathrm{~d}$ time points. (D-F). Down-regulated DEGs at $6 \mathrm{~h}, 48 \mathrm{~h}, 8 \mathrm{~d}$ time points. Statistically significant DEGs were defined with $\mathrm{p}<0.05$ and logFC $>2$ as the cut-off criterion (fold change, FC). 


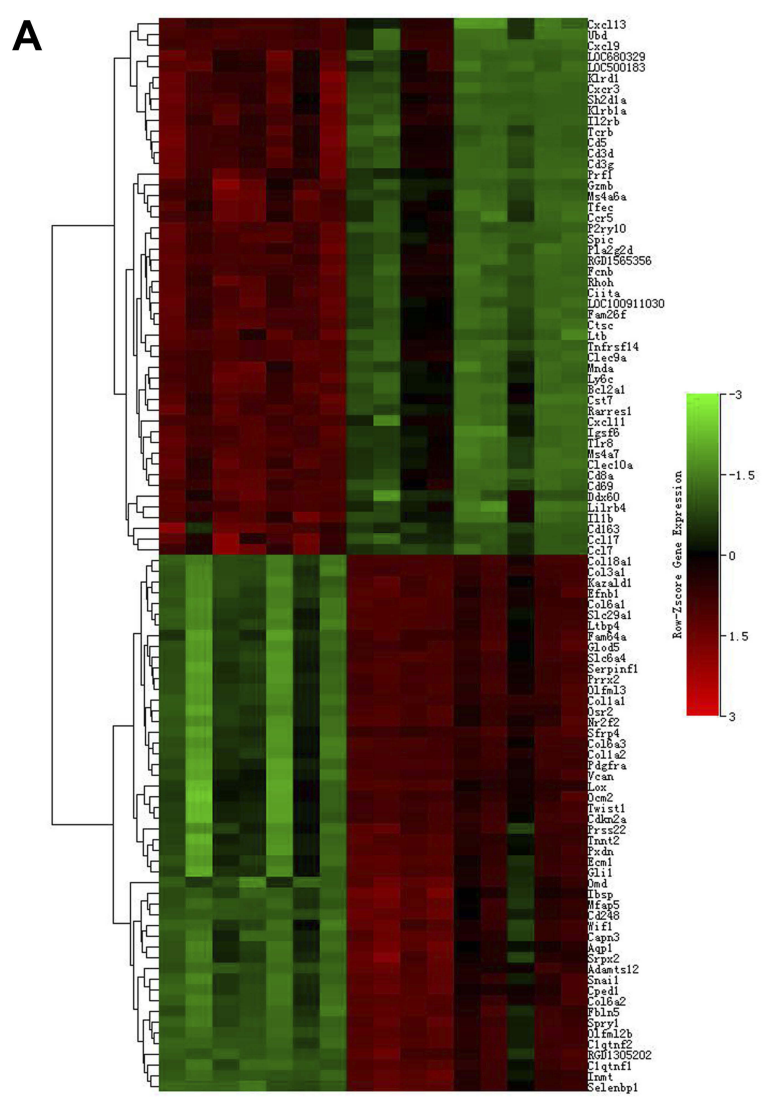

MRT vs untreated glioma
B

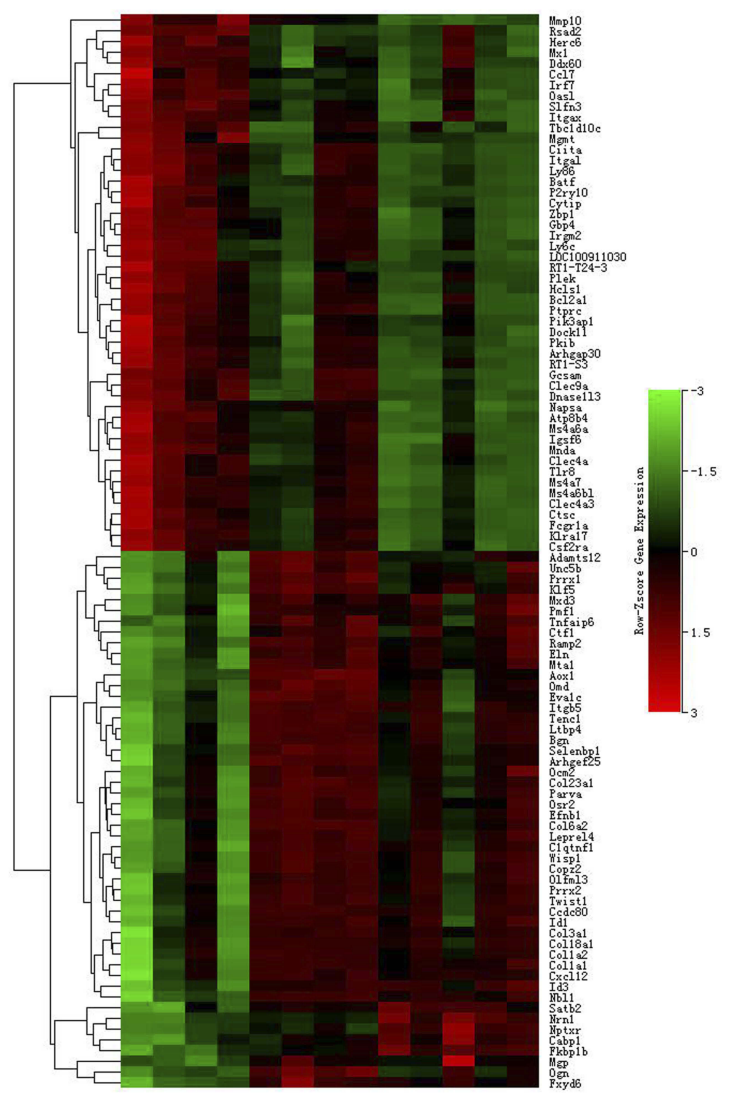

BB vs untreated glioma

\section{C}

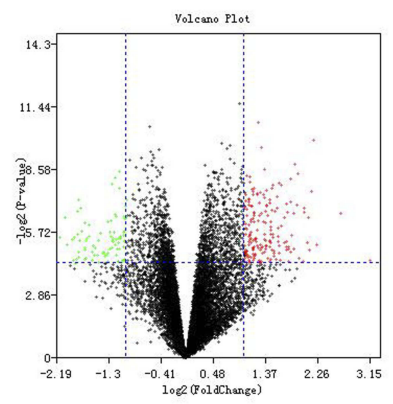

MRT vs BB

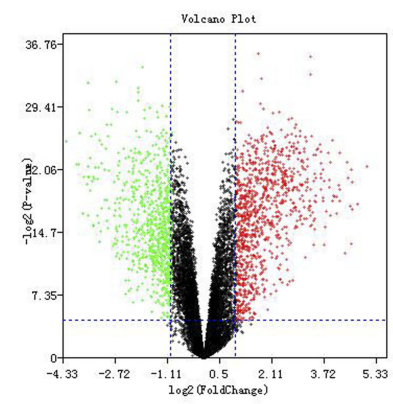

MRT vs untreated glioma

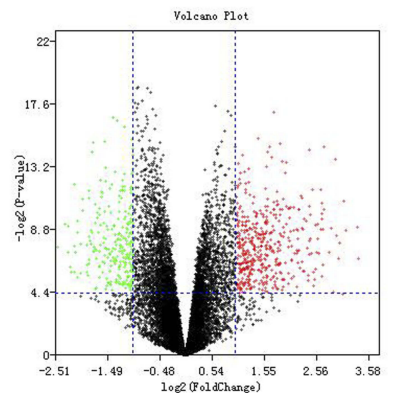

BB vs untreated glioma

Figure 2 (A). Heat map showing gene expression profiles of MRT (Synchrotron microbeam radiation therapy) therapy-treated glioma tissues, as well as gene enrichment analysis. X-axis indicated samples, and Y-axis indicated genes. (B). Heat map showing gene expression profiles of BB (broad-beam) therapy-treated glioma tissues, as well as gene enrichment analysis. (C). Volcano plot of the aberrantly expressed genes of MRT vs BB; MRT vs untreated; BB vs untreated glioma tissues.

cell surface, immunological synapse, extracellular space, and integral component of the membrane. In the molecular function group, upregulated genes were mainly enriched in chemokine activity, heparin-binding, and carbohydrate-binding. The downregulated genes were mainly enriched in elastic fiber, extracellular space, and matrix.

Regarding signaling pathway enrichment analysis, upregulated genes were mainly enriched in primary immunodeficiency, cytosolic DNA-sensing pathway, and Toll-like receptor (TLR) signaling pathway. Downregulated genes were mainly enriched in protein digestion and absorption, ECM-receptor interaction, and focal adhesion (Table 2).

\section{PPI Network and Modular Analysis}

A total of 104 upregulated DEGs and 13 downregulated DEGs obtained from the Venn plot (Figure 1) were imported into the DEGs PPI network complex with an edge score $>0.4$ (Figure $3 \mathrm{~A}, \mathrm{~B}$ ). To identify the hub 
Table I Gene Ontology (GO) Analysis and Significant Enriched GO Terms of Differentially Expressed Genes (Absolute Log2 Fold Change $(\mathrm{FC})>2$ ) Based on Their Functions in Murine Glioblastoma After Radiotherapy (i.e. Molecular Function, Biological Process and Cellular Component)

\begin{tabular}{|c|c|c|c|c|c|}
\hline GOTERM_BP_DIRECT & GO:005 I 607 Defense Response to Virus & 10 & 0.09738 & I.27E-08 & I.89E-05 \\
\hline GOTERM_BP_DIRECT & GO:0006954 inflammatory response & 13 & 0.126595 & $2.23 \mathrm{E}-08$ & 3.33E-05 \\
\hline GOTERM_BP_DIRECT & GO:0070098 chemokine-mediated signaling pathway & 7 & 0.068166 & $3.46 \mathrm{E}-07$ & $5.17 E-04$ \\
\hline GOTERM_BP_DIRECT & GO:0006955 immune response & II & 0.107119 & $7.25 \mathrm{E}-07$ & 0.001082 \\
\hline GOTERM_BP_DIRECT & GO:00302I $7 \sim T$ cell differentiation & 6 & 0.058428 & $1.65 \mathrm{E}-06$ & 0.002456 \\
\hline GOTERM_BP_DIRECT & GO:0032496 response to lipopolysaccharide & 10 & 0.09738 & $9.32 \mathrm{E}-06$ & 0.013912 \\
\hline GOTERM_BP_DIRECT & GO:007I346 cellular response to interferon-gamma & 6 & 0.058428 & I.2IE-05 & 0.01805 \\
\hline GOTERM_BP_DIRECT & GO:0045087 innate immune response & 9 & 0.087642 & $4.22 \mathrm{E}-05$ & 0.062903 \\
\hline GOTERM_BP_DIRECT & GO:0010332 response to gamma radiation & 5 & 0.04869 & $7.52 \mathrm{E}-05$ & 0.112185 \\
\hline GOTERM_CC_DIRECT & GO:0009897 external side of plasma membrane & 13 & 0.126595 & $8.21 \mathrm{E}-09$ & 8.7IE-06 \\
\hline GOTERM_CC_DIRECT & GO:0009986 cell surface & 13 & 0.126595 & 4.95E-05 & 0.052459 \\
\hline GOTERM_CC_DIRECT & GO:000 I772 immunological synapse & 3 & 0.029214 & 0.012351 & 12.3475 \\
\hline GOTERM_CC_DIRECT & GO:0005615 extracellular space & 14 & 0.136333 & 0.012996 & 12.95267 \\
\hline GOTERM_CC_DIRECT & GO:0016021 integral component of membrane & 37 & 0.360308 & 0.055425 & 45.37502 \\
\hline GOTERM_CC_DIRECT & GO:0008305 integrin complex & 2 & 0.019476 & 0.094847 & 65.24212 \\
\hline GOTERM_MF_DIRECT & GO:0008009 chemokine activity & 5 & 0.048690233 & $2.59 \mathrm{E}-05$ & $0.03 \mid 4575$ \\
\hline GOTERM_MF_DIRECT & GO:000820I heparin binding & 6 & 0.058428279 & $6.86 \mathrm{E}-04$ & 0.8306152 \\
\hline GOTERM_MF_DIRECT & GO:0030246 carbohydrate binding & 6 & 0.058428279 & 0.00112535 & 1.3588387 \\
\hline GOTERM_MF_DIRECT & GO:0005525 GTP binding & 7 & 0.068166326 & 0.00989358 & 11.380067 \\
\hline GOTERM_MF_DIRECT & GO:0003924 GTPase activity & 5 & 0.048690233 & 0.01520823 & 16.990193 \\
\hline \multicolumn{6}{|l|}{ Down-Regulated } \\
\hline GOTERM_CC_DIRECT & GO:007/953 elastic fiber & 2 & 0.103093 & 0.00201 & I.24577| \\
\hline GOTERM_CC_DIRECT & GO:00056I5 extracellular space & 3 & 0.154639 & 0.05895 & 31.51105 \\
\hline GOTERM_CC_DIRECT & GO:0005578 proteinaceous extracellular matrix & 2 & 0.103093 & 0.078698 & 39.98713 \\
\hline GOTERM_CC_DIRECT & GO:007I 953 elastic fiber & 2 & 0.103093 & 0.00201 & I.24577| \\
\hline GOTERM_CC_DIRECT & GO:0005615 extracellular space & 3 & 0.154639 & 0.05895 & 31.51105 \\
\hline
\end{tabular}

Abbreviation: FDR: false discovery rate.

genes, the Cytotype MCODE plug-in was applied for further analysis and 19 core nodes of upregulated genes and 6 genes of downregulated genes were identified (Figure 3C, D).

\section{Analysis of Core Genes in Function and Pathway Enrichment}

Both GO and KEGG were searched for core genes including upregulated and downregulated genes. Pathway analysis results are shown in Figure 4A; count represents the gene number and while-log (p-value) represents the significance of gene enrichment. The more-log (p-value) represents greater significance. Upregulated genes were mainly enriched in tuberculosis, cytokine-cytokine receptor signaling, chemokine signaling pathway, and TLR signaling pathway. Downregulated genes were mainly enriched in protein digestion and absorption, ECM-receptor interaction, and focal adhesion (Figure 4B).

GO analysis of the DEGs in the biological process group showed upregulated genes were mainly enriched in positive regulation of natural killer cell chemotaxis such as phagocytosis recognition and positive regulation of IL-6 biosynthetic processes. Regarding DEGs in the cellular component group, upregulated genes were mainly enriched in integrin complex, external side of plasma membrane, and extracellular space. Regarding DEGs in the molecular function group, upregulated genes were mainly enriched in transmembrane signaling receptor activity (Figure 4C). Downregulated genes were mainly enriched in elastic fiber production (Figure 4D, cellular component). The values in Figure $4 \mathrm{~A}$ and $\mathrm{B}$ were the number of genes. Count represented the genes number, while $-\log (\mathrm{P}$ value) represented the significance of gene enrichment. The more $\log (\mathrm{P}$ value) was, the higher significance it represented. For Figure $4 \mathrm{C}$ and $\mathrm{D}$, the value was fold enrichment score.

\section{Re-Analysis of Core Genes Using the Kaplan-Meier Plotter}

Gene prognosis analysis was conducted using UALCAN (http://ualcan.path.uab.edu/). For Figure 5 they were the 
Table 2 KEGG Pathways of Differentially Expressed Genes After Radiotherapy

\begin{tabular}{|c|c|c|c|c|}
\hline Up-Regulated Expession Category Term & Count & $\%$ & P-value & FDR \\
\hline rno05340:Primary immunodeficiency & 6 & 0.05842828 & $2.49 \mathrm{E}-06$ & 0.002676258 \\
\hline rno04640:Hematopoietic cell lineage & 9 & 0.08764242 & $2.50 \mathrm{E}-08$ & $2.69 \mathrm{E}-05$ \\
\hline rno04623:Cytosolic DNA-sensing pathway & 5 & 0.04869023 & $3.46 \mathrm{E}-04$ & 0.372044097 \\
\hline rno04620:Toll-like receptor signaling pathway & 7 & 0.06816633 & $2.34 \mathrm{E}-05$ & 0.025133454 \\
\hline rno05332:Graft-versus-host disease & 4 & 0.03895219 & 0.007325943 & 7.605983524 \\
\hline rno04060:Cytokine-cytokin receptor interaction & 12 & 0.11685656 & $1.89 \mathrm{E}-08$ & $2.03 \mathrm{E}-05$ \\
\hline rno05323:Rheumatoid arthritis & 5 & 0.04869023 & 0.002073222 & 2.208095724 \\
\hline rno05I50:Staphylococcus aureus infection & 3 & $0.0292|4| 4$ & 0.042201732 & 37.11710221 \\
\hline rno04940:Type I diabetes mellitus & 4 & 0.03895219 & 0.010790373 & 11.01670867 \\
\hline rno0462I:NOD-like receptor signaling pathway & 3 & $0.0292|4| 4$ & 0.046534697 & 40.11101512 \\
\hline rno04650:Natural killer cell-mediated cytotoxicity & 5 & 0.04869023 & 0.002829197 & 3.002182187 \\
\hline rno05I52:Tuberculosis & 9 & 0.08764242 & I.I4E-05 & 0.012270825 \\
\hline rno04660:T cell receptor signaling pathway & 5 & 0.04869023 & 0.003630094 & 3.837070925 \\
\hline rno04666:Fc gamma R-mediated phagocytosis & 4 & 0.03895219 & $0.0145973 \mid 4$ & 14.63261537 \\
\hline rno04062:Chemokine signaling pathway & 8 & 0.07790437 & $8.38 \mathrm{E}-05$ & 0.090088077 \\
\hline rno04064:NF-kappa B signaling pathway & 4 & 0.03895219 & 0.016504231 & 16.39326904 \\
\hline rno05416:Viral myocarditis & 4 & 0.03895219 & 0.017001852 & 16.84726968 \\
\hline rno05330:Allograft rejection & 3 & $0.0292|4| 4$ & 0.067034727 & 52.59863562 \\
\hline rno05 I40:Leishmaniasis & 3 & $0.0292|4| 4$ & $0.0704 I 5454$ & $54.4|428| 13$ \\
\hline \multicolumn{5}{|l|}{ Down-Regulated } \\
\hline cfa04974:Protein digestion and absorption & 4 & 0.206185567 & $1.86 \mathrm{E}-06$ & $8.90 \mathrm{E}-04$ \\
\hline cfa045 I 2:ECM-receptor interaction & 2 & 0.103092784 & 0.03817602 & 16.99625568 \\
\hline cfa045 I0:Focal adhesion & 2 & 0.103092784 & 0.089220317 & 36.0622488 \\
\hline
\end{tabular}

prognostic information of the 25 core genes which were identified with Cytoscape software (for protein-protein interaction and hub genes). The results showed that 19 central nodes of up-regulated genes and 6 genes of downregulated genes which were identified.

Among the 25 core genes, two genes (Ccl7 and CXCR3) correlated with significant poor prognosis $(\mathrm{P}<0.05)$ and the other 23 genes had no significant correlation $(\mathrm{P}>0.05)$, the genes are shown in Table 3.

\section{Gene Expressions Compared Between Tumor Tissues and Contralateral Normal Brain Tissues}

The function and pathway enrichment analysis of all DEGs and core DEGs showed the changes mainly occurred in immune responses, especially chemotaxis and the IL-6 signaling pathway (Figure 4). To determine whether the immune responses were due to tumoral antigens released by radiationinduced apoptosis, the DEGs were compared between tumor tissues and contralateral normal brain tissues $8 \mathrm{~d}$ after radiation. The heatmap is shown in Figure 6A. The heatmap regarding chemotaxis and IL-6-associated genes is shown in Figure 6B.
Chemotaxis and IL-6-associated genes were significantly more upregulated in tumor tissues than in normal brain tissues.

Radiation increased chemotaxis and IL-6-associated genes in GBM cell strain U87. Chemotaxis and IL-6-associated genes were examined in U87 cells to further verify the upregulation in GBM cells. Real-time PCR was performed and GAPDH used as the reference gene. The gene expressions were normalized as 1 before irradiation. (GAPDH was used as reference gene)

Usually, the number of cells will decrease on the first day after cell subculture. We chose $10^{\wedge} 6$ of U87 cells to seed in $75 \mathrm{~cm} 2$ flasks, after 1 day of the latency period, the cells will go into logarithmic phase and after $48 \mathrm{~h}$ the cells will go into $100 \%$ confluence rate stage. In logarithmic phase, cells grow fast and show strong viability. When the cells are at $100 \%$ confluence rate stage, we start radiation and obvious cell death will happen. A certain number of the residual alive cells will guarantee cell touchment from each other and guarantee survival rate. This also guarantees the possibility of the survival of radioresistant cells.

As shown in Figure 7, Ccl-2, Ccl-5, Ccl-7, TLR1, IL1B, and IL- 6 were examined before and after radiation. All the genes were significantly upregulated $(\mathrm{P}<0.05)$ when 
A

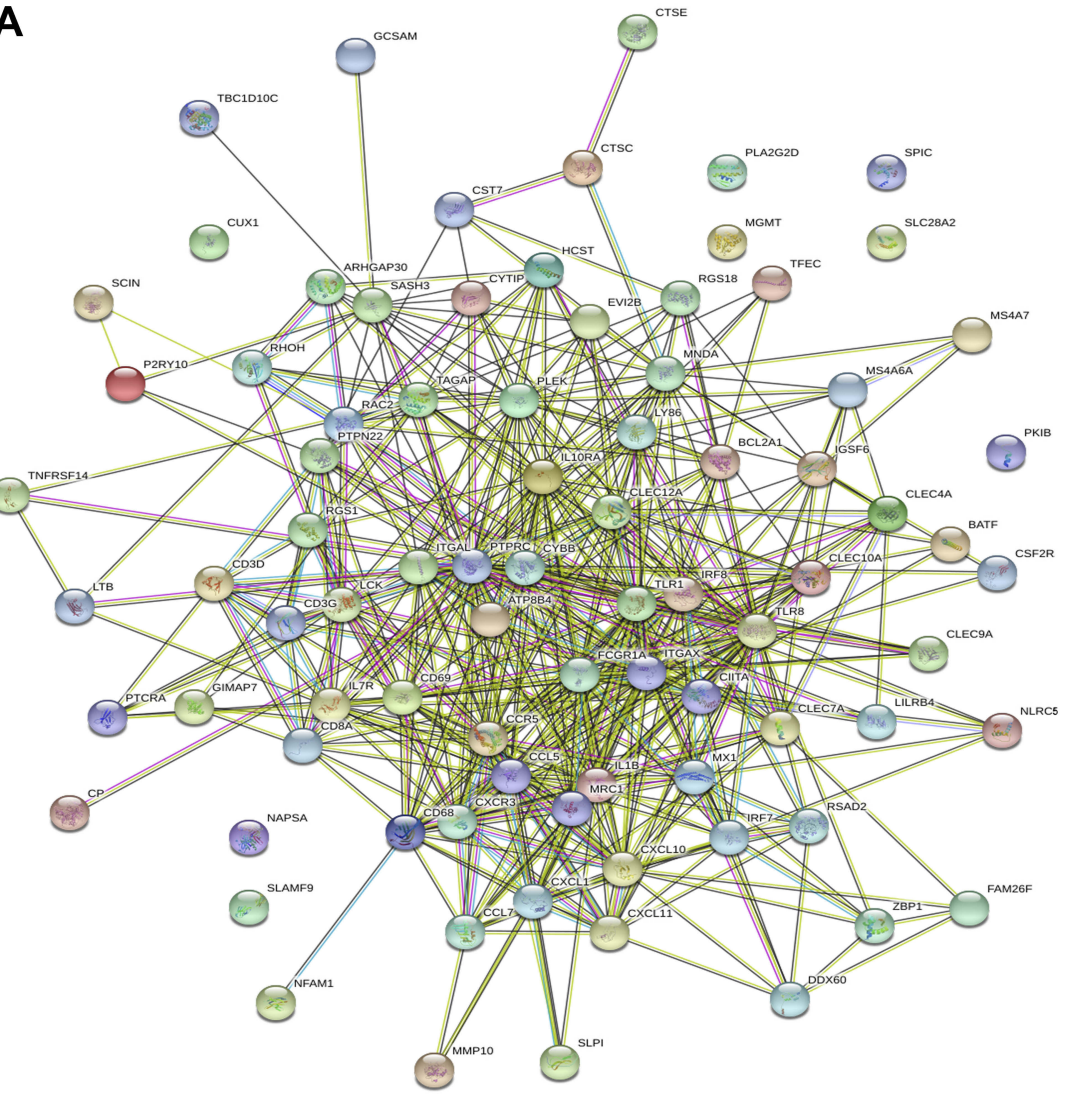

C

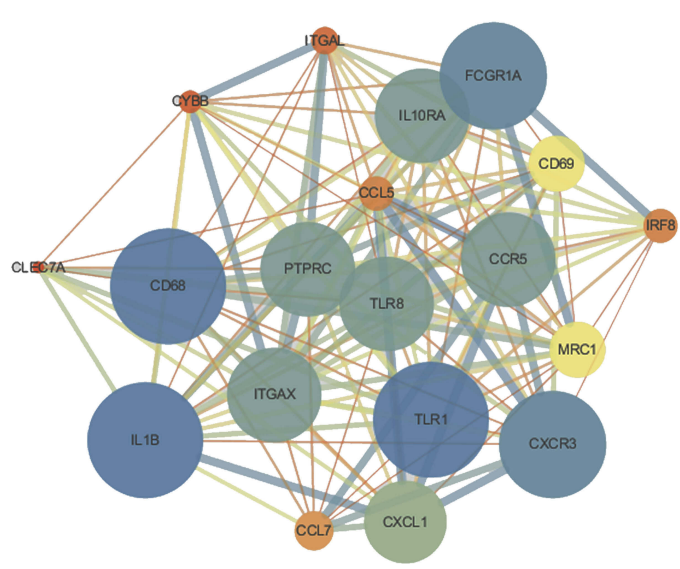

B
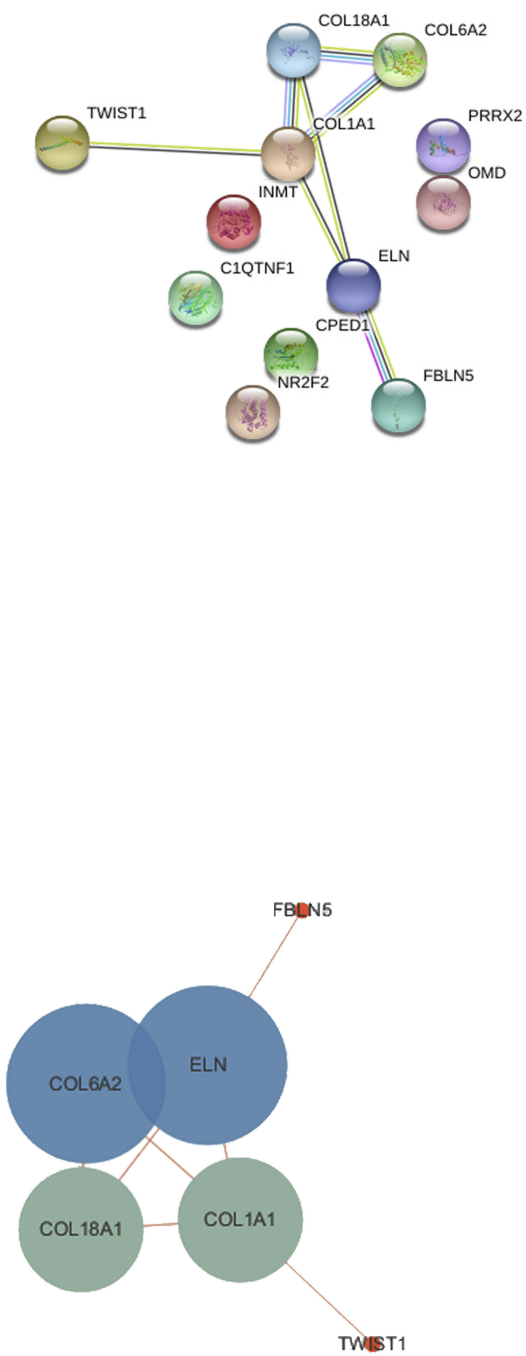

Figure 3 Construction of common DEGs (differentially expressed genes) PPI (protein-protein interaction) network by STRING online database (http://string-db.org/) and Module analysis for up-regulated (A) and down-regulated (B) genes. There were a total of 117 DEGs (differentially expressed genes) in the network complex. The nodes represented proteins and the edges represented the interaction score of proteins. (C, D). Module analysis via Cytoscape software -19 up-regulated core genes (C) and 6 down-regulated core genes (D) were selected.

exposed 3 times to 1 Gy irradiation, 3 times to 2 Gy irradiation, and 7 times to 4 Gy irradiation.

\section{Discussion}

GBM is a type of brain tumor with poor prognosis. ${ }^{16}$ Numerous basic and clinical studies have been conducted to determine the cause of GBM relapse in the past several decades. ${ }^{17,18}$ Major improvements in the survival outcomes of patients with GBM have not been achieved in recent years. Due to the high aggressiveness and low metastasis ability, radiotherapy became the primary treatment for GBM. ${ }^{19,20}$ Nevertheless, GBM is strongly resistant to chemotherapy and radiotherapy. ${ }^{21}$ However, due to ethical limitations, human post-radiotherapy GBM samples can rarely be obtained. Murine GBM models were constructed to simulate and substitute the human in vivo 
A

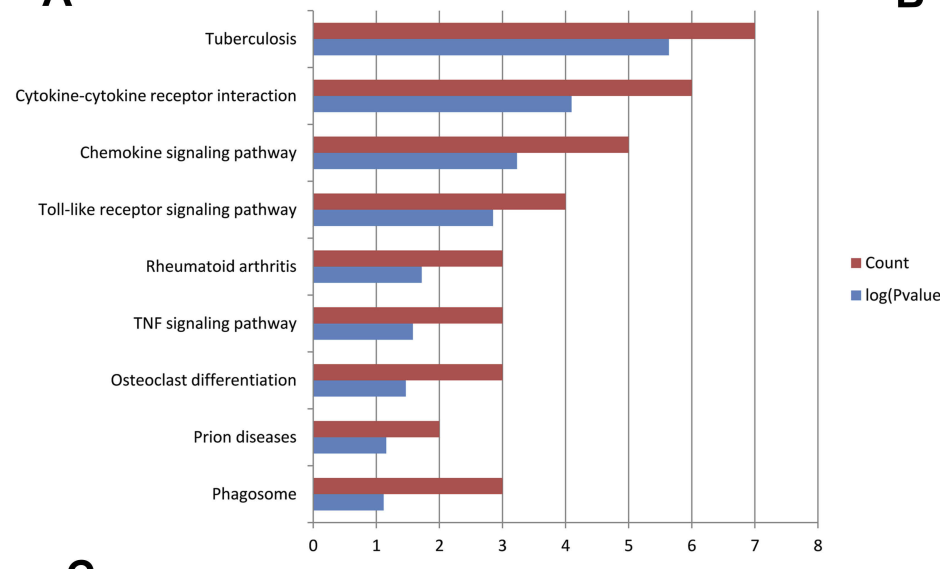

C

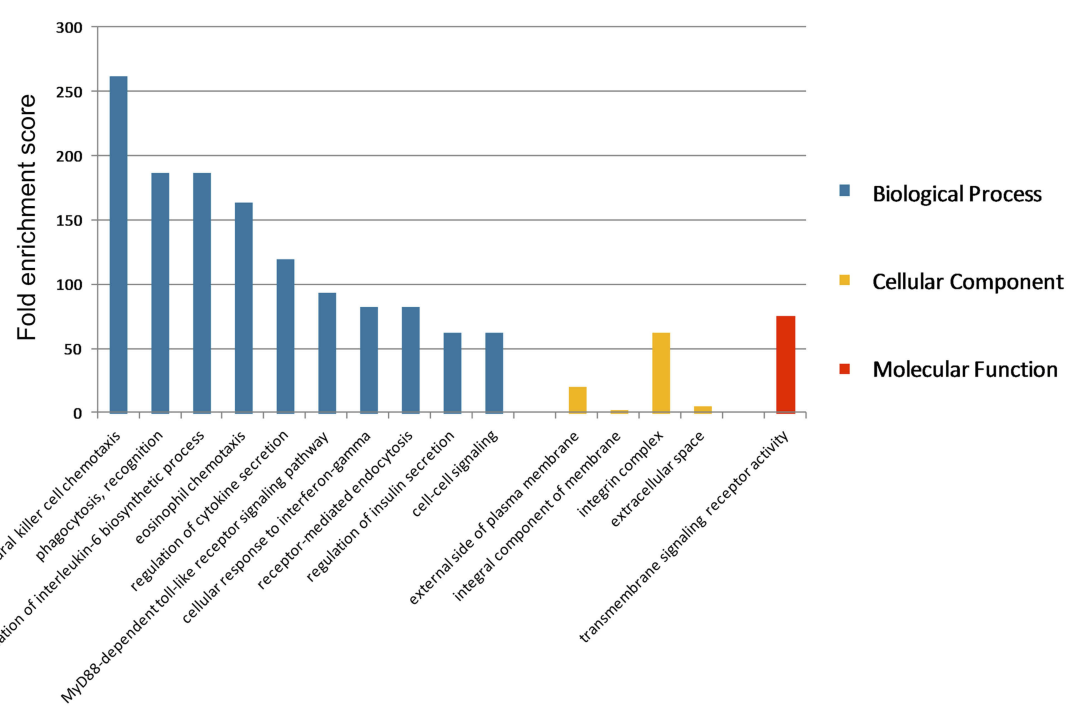

B

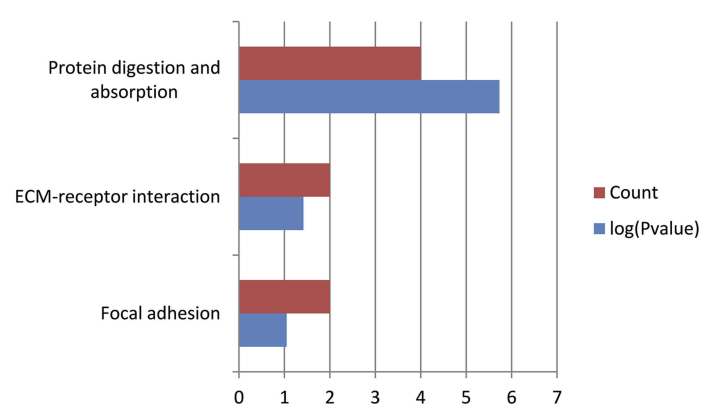

D

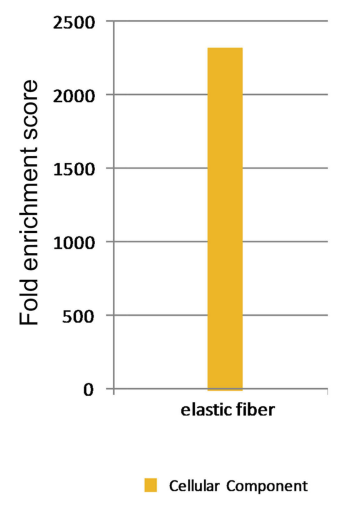

Figure 4 Significant enriched Kyoto Encyclopedia of Genes and Genomes (KEGG) pathways of core differentially expressed genes (A, B) and biological processes, cellular component, molecular function of gene ontology (GO) analysis (C, D) to predict the potential roles of identified core DEGs (differentially expressed genes). Count represented the genes number, while - $\log (\mathrm{P}$ value) represented the significance of gene enrichment. (A). KEGG analysis for up-regulated genes. (B). KEGG analysis for down-regulated genes. (C). GO analysis for up-regulated genes. (D). GO analysis for down-regulated genes.

Abbreviations: TNF, tumor necrosis factor; ECM, extracellular matrix; MyD88, myeloid differentiation factor 88.

environment. In the present study, a murine microarray RNA profile dataset was integrated from different treatment methods. Bioinformatics was used to thoroughly analyze the high-throughput data and 117 significantly changed DEGs (104 upregulated and 13 downregulated) were identified $8 \mathrm{~d}$ after radiotherapy.

The DEGs were further clustered based on functions and signaling pathways and enrichment analysis performed. Next, the DEGs PPI network complex was developed and the core gene module was selected using Cytotype MCODE analysis.

Notable variances in the aberrant gene profiling and molecular characteristics between tumor tissues and contralateral normal brain tissues were observed, which resulted in the different microenvironments after radiotherapy. The core gene module obtained from PPI analysis was used to search the KEGG and GO databases. Consequently, the core DEGs were particularly enriched in chemotaxis and the IL-6 signaling pathway which are involved in the immune response. Downregulated DEGs were enriched in extracellular matrix production. The reason for radiotherapy failure may partially depend on the change in the immune microenvironment. Among the pathways, chemokine signaling and IL-6 signaling pathways play an important role in the immune response, which was confirmed in human radioresistant cell strains.

The immune response is closely associated with tumor proliferation, migration, and anti-apoptotic abilities. $^{22}$ GBM rarely metastasizes to extracranial $\operatorname{sites}^{23}$ although tumor cells have been found in the 

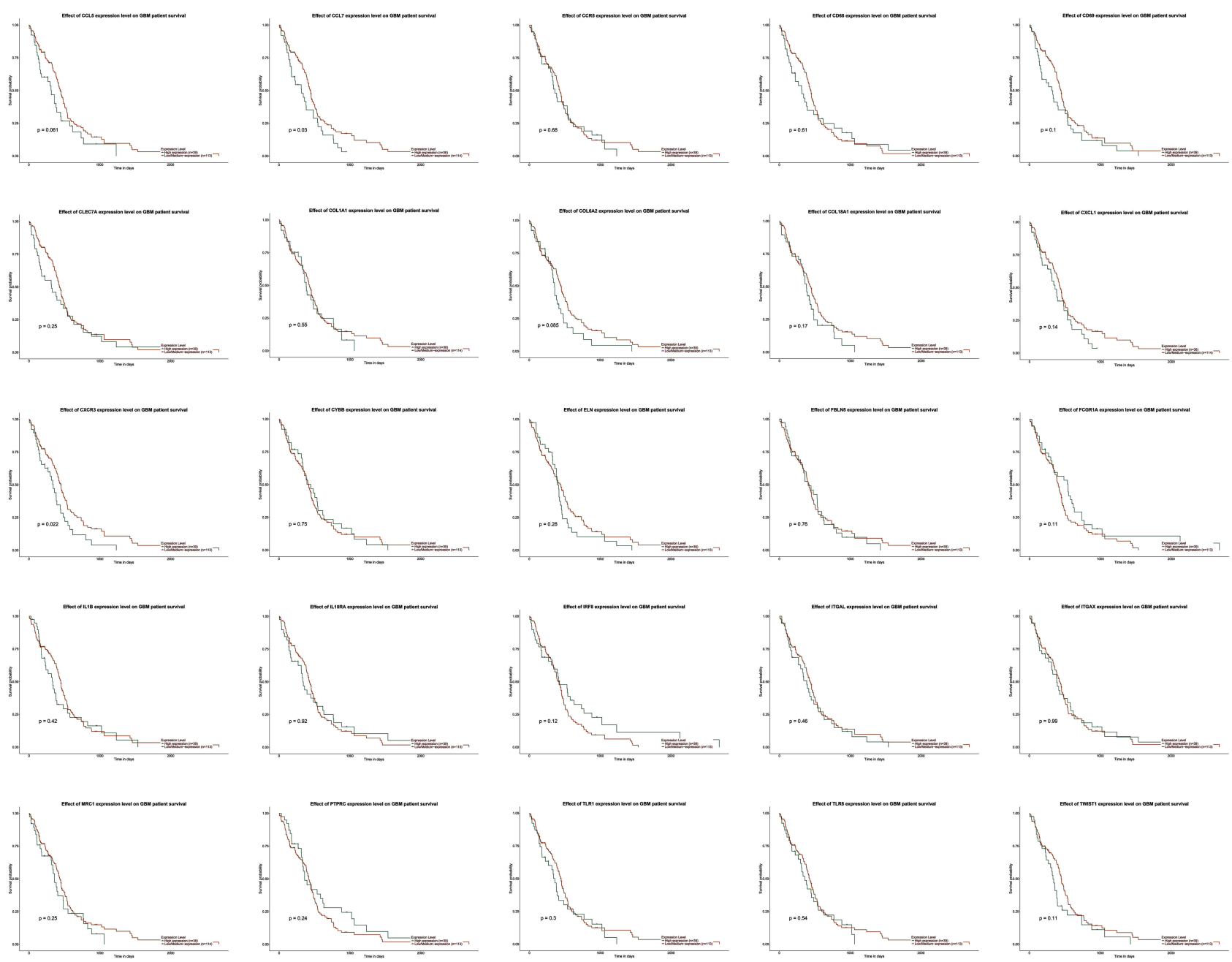

Figure 5 The prognostic information of the 25 core genes identified from Cytoscape software (19 up-regulated core genes and 6 down-regulated core genes). Kaplan Meier plotter online tool UALCAN (http://ualcan.path.uab.edu/) was used to identify the prognostic information of the genes. Among the 25 core genes, 2 core genes had a significantly poor survival rate $(\mathrm{P}<0.05)$.

Abbreviations: GBM, glioblastoma; CCL5, chemokine C-C motif ligand 5; CCL7, chemokine C-C motif ligand 7; CCR5, C-C chemokine receptor type 5; CD68, cluster of differentiation 68; CD69, cluster of differentiation 69; CLEC\&A, C-type lectin domain family 7, member A; COLIAI, Collagen Type I Alpha I; COL6A2, collagen, type VI, alpha 2; COLI8AI, collagen, type XVIII, alpha I; CXCLI, C-X-C motif chemokine ligand I; CXCR3, C-X-C chemokine receptor type 3; CYBB, cytochrome b-245, beta chain; ELN, elastin; FBLN5, fibulin-5; FCGRIA, high affinity immunoglobulin gamma Fc receptor I; ILIB, interleukin I, beta; ILIORA, Interleukin-I0 receptor subunit alpha; IRF8, Interferon regulatory factor 8; ITGAL, Integrin alpha-L; ITGAX, integrin alpha X; MRCI, macrophage mannose receptor I; PTPRC, protein tyrosine phosphatase, receptor type, C; TLRI, toll-like receptor I; TLR8, toll-like receptor 8; TWISTI, twist-related protein I.

peripheral circulation system. ${ }^{24}$ Therefore, GBM cells were considered fragile and could barely survive in extracranial sites due to the rapid colonization of the tumor inside the brain. Systemic immunosuppression has been demonstrated in patients with GBM. ${ }^{25}$ The mechanisms underlying the immune suppression effects require further exploration; however, both tumorintrinsic factors and host responses to tumor antigens originating from GBM may be involved in the process. ${ }^{26}$

The IL-6 signaling pathway has been studied in several types of cancer. ${ }^{27}$ IL-6-activated JAK1 phosphorylates programmed death-ligand 1 (PD-L1) Tyr112 to catalyze PD-L1 glycosylation and maintain PD-L1 stability in hepatocellular carcinoma; thus, targeting IL-6 could prevent cancer immune evasion. ${ }^{28}$ IL-6 could also affect monocytes and turn them into monocytic tumor-associated macrophage-like cells which induce an immunosuppressive microenvironment. ${ }^{29}$ Endoplasmic reticulum stress can induce radioresistance through the IL-6 pathway in oropharyngeal carcinoma. ${ }^{30}$ Gamma-irradiation and some chemotherapy drugs such as 5-Fu can induce production of IL-6 and IL-8 providing a potential target for radiochemosensitization. ${ }^{31}$ IL-6 signaling also contributes to radioresistance of prostate cancer through 
Table 3 The Prognostic Information of the 20 Key Candidate Genes

\begin{tabular}{|c|c|c|c|c|c|}
\hline Category & Genes & & & & \\
\hline $\begin{array}{l}\text { Genes with } \\
\text { significantly } \\
\text { worse survival } \\
(P<0.05)\end{array}$ & CCL7 & $C X C R 3$ & & & \\
\hline $\begin{array}{l}\text { Genes without } \\
\text { significantly CCL5 }\end{array}$ & COL6A2 & CCR5 & CD68 & CD69 & \\
\hline \multirow[t]{2}{*}{$\begin{array}{l}\text { worse survival } \\
(P>0.05)\end{array}$} & CLEC7A & CXCLI & $C Y B B$ & FCGRIA & \\
\hline & $\begin{array}{l}I L I B \\
M R C I\end{array}$ & $\begin{array}{l}\text { ILIORA } \\
\text { PTPRC }\end{array}$ & $\begin{array}{l}\text { IRF8 } \\
\text { TLRI }\end{array}$ & $\begin{array}{l}\text { IGTAL } \\
\text { TLR8 }\end{array}$ & IGTAX \\
\hline
\end{tabular}

Note: Two genes ( $C \mathrm{Cl}-7$ and $C X C R 3)$ had a significantly poor survival rate $(P<0.05)$.

key DNA repair-associated molecules such as BRCA $1 / 2{ }^{32}$ Repression of IL-6 increased chemosensitivity of cisplatin in esophageal squamous cell carcinoma via the STAT3 pathway. ${ }^{33}$ IL-1B is a major IL-6 activator and works together with IL-6 to provide the commitment signal for IL-10-secreting regulatory B cells. ${ }^{34}$
TLR 1 participates in the induction of IL- 6 and recruitment of TGF- $\beta$ in the intestinal environment. ${ }^{35}$

Chemokines such as Ccl-2, Ccl-5, and Ccl-7 were also upregulated in irradiated tumor tissues. Ccl-7 receptor CCR7 reportedly is correlated with metastasis in nasopharyngeal carcinoma. ${ }^{36}$ Radiotherapy upregulated CCL2 chemokine production in tumor cells leading to a CCR2-dependent accumulation of tumor necrosis factor alpha (TNF $\alpha$ )-producing monocytes and CCR2+ regulatory $\mathrm{T}$ cells (Tregs), thus, leading to radioresistance. ${ }^{37}$ Chemokine receptor CXCR3 is involved in lymph node metastasis ${ }^{38}$ and was one of the 19 upregulated core genes in the present study.

Gene expression profiles after radiotherapy comprehensively affect the treatment. The crosstalk between tumor and immune microenvironment has previously been investigated. ${ }^{39,40}$ However, studies based on highthroughput RNA data after radiotherapy have rarely been reported. Therefore, the novel results from the present study could provide information and direction for future GBM cancer studies. Immune-associated treatment against
A

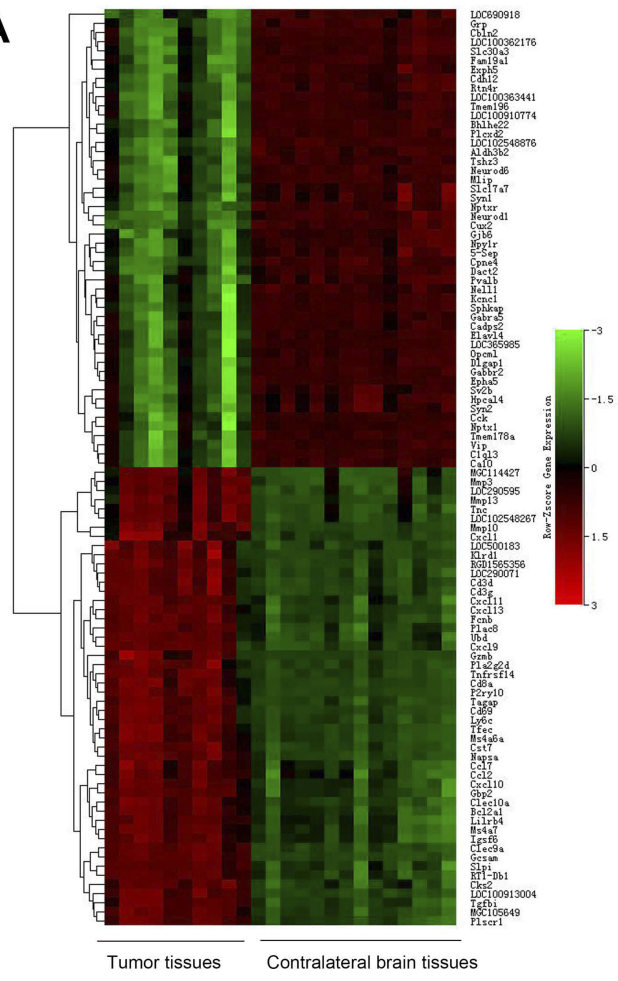

\section{B}

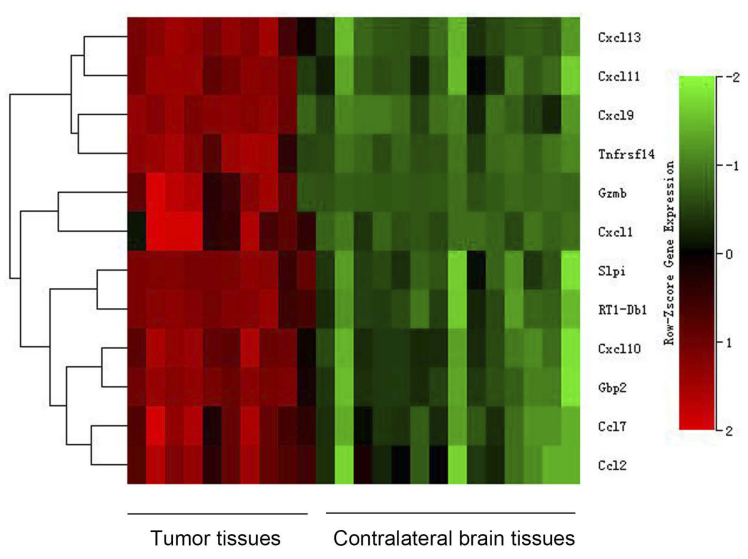

Figure 6 Heat map showing gene expression profiles of radiated tumor tissues and contralateral normal brain tissues (A). Chemotaxis pathway and IL-6 (interleukin-6) signaling pathway-related genes between radiated tumor tissues and contralateral normal brain tissues (B). 
A

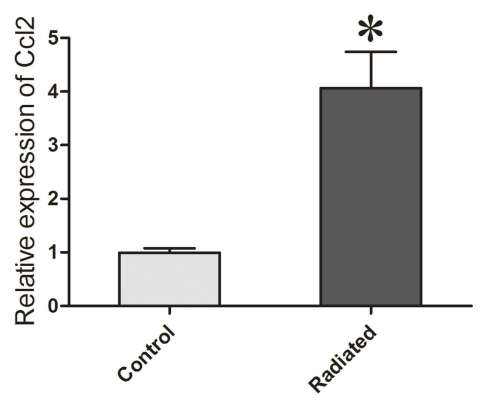

D

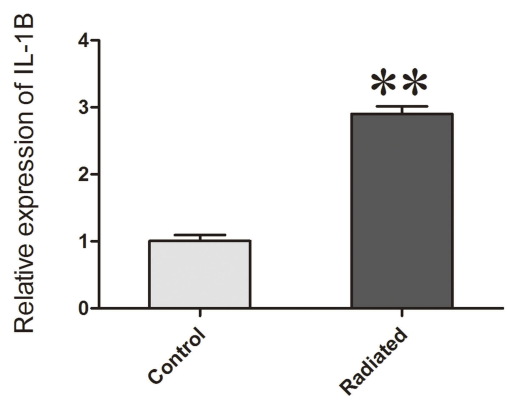

B

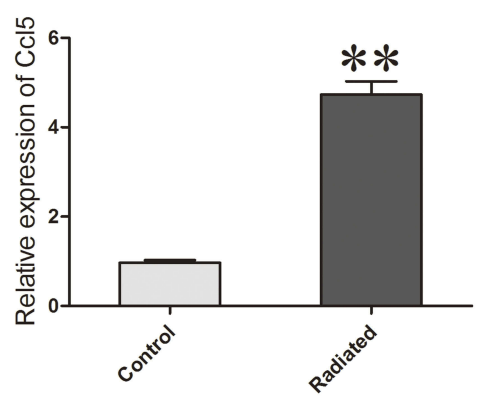

E

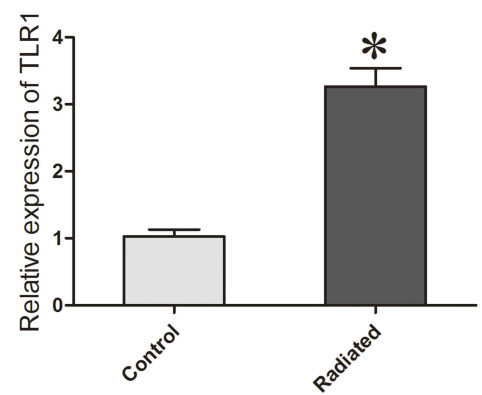

C

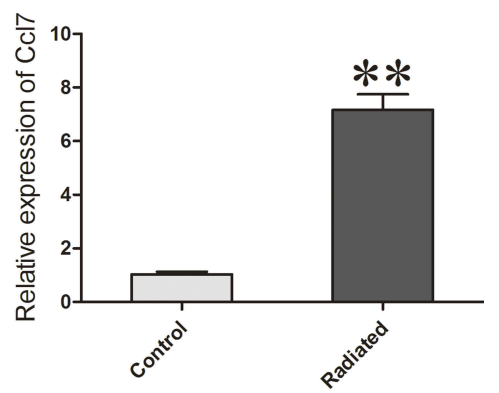

$\mathbf{F}$

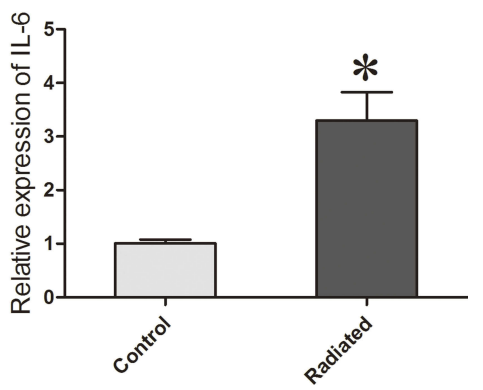

Figure 7 Ccl-2 (Chemokine (C-C motif) ligand 2) (A), Ccl-5 (Chemokine (C-C motif) ligand 5) (B), Ccl-7 (Chemokine (C-C motif) ligand 7) (C), TLR I (Toll-like receptor I) (D), IL-IB (interleukin-IB) (E) and IL-6 (interleukin-6) (F) expression changes between untreated U87 cells and radioresistant U87 cells. The mRNA expression level of all genes was normalized against the endogenous control of GAPDH (glyceraldehyde-3-phosphate dehydrogenase). $(n=3, * P<0.05, * * P<0.0 I)$.

IL-6 and chemokine signaling pathway may be applicable to radiotherapy; however, further investigation is needed.

\section{Abbreviations}

GBM, Glioblastoma; DAVID, Database for Annotation, Visualization and Integrated Discovery; BBB, bloodbrain barrier; DEGs, differentially expressed genes; GEO, Gene Expression Omnibus database; MCODE, Molecular Complex Detection; GO, Gene Ontology database; MRT, microbeam radiation therapy; BB, broad-beam therapy; PPI, protein-protein interaction network; PD-L1, programmed death-ligand 1; TLR, Toll-like receptor.

\section{Acknowledgments}

This study was funded by the National Natural Science Foundation of China (No. 81702402, No. 81802760), Science and Technology Project of Liaoning (No. 20170520027).

\section{Disclosure}

The authors report no conflicts of interest in this work. All authors claimed that there was no such things as consultancies, honoraria, stock ownership, gifts, free or reimbursed travel/vacations, equity interests, arrangements regarding patents or other vested interests, etc. No financial relationships with any pharmaceutical company.

\section{References}

1. Van Meir EG, Hadjipanayis CG, Norden AD, Shu HK, Wen PY, Olson JJ. Exciting new advances in neuro-oncology: the avenue to a cure for malignant glioma. CA Cancer J Clin. 2010;60:166-193. doi:10.3322/caac.20069

2. Perry JR, Laperriere N, Mason WP. Radiation plus temozolomide in patients with glioblastoma. $N$ Engl J Med. 2017;376:2197. doi:10.1056/NEJMoa1611977

3. Omuro A, DeAngelis LM. Glioblastoma and other malignant gliomas: a clinical review. JAMA. 2013;310(17):1842-1850. doi:10.1001/ jama.2013.280319

4. Sulman EP, Ismaila N, Chang SM. Radiation therapy for glioblastoma: American Society of Clinical Oncology Clinical Practice Guideline Endorsement of the American Society for Radiation Oncology Guideline. J Oncol Pract. 2017;13:123-127. doi:10.1200/JOP.2016.018937

5. Herrera FG, Bourhis J, Coukos G. Radiotherapy combination opportunities leveraging immunity for the next oncology practice. $C A$ Cancer J Clin. 2017;67:65-85. doi:10.3322/caac.21358

6. Lim M, Xia Y, Bettegowda C, Weller M. Current state of immunotherapy for glioblastoma. Nat Rev Clin Oncol. 2018;15(7):422-442. doi:10.1038/s41571-018-0003-5

7. Jiang H, Chess L. An integrated view of suppressor T cell subsets in immunoregulation. J Clin Invest. 2004;114(9):1198-1208. doi: $10.1172 / \mathrm{JCI} 23411$

8. McGee HM, Jiang D, Soto-Pantoja DR, Nevler A, Giaccia AJ, Woodward WA. Targeting the tumor microenvironment in radiation oncology: proceedings from the 2018 ASTRO-AACR Research Workshop. Clin Cancer Res. 2019;25:2969-2974. doi:10.1158/10780432.CCR-18-3781 
9. Billingham RE, Brent L, Medawar PB, Sparrow EM. Quantitative studies on tissue transplantation immunity. I. The survival times of skin homografts exchanged between members of different inbred strains of mice. Proc R Soc Lond B Biol Sci. 1954;143:43-58.

10. Alvarez JI, Dodelet-Devillers A, Kebir H, et al. The Hedgehog pathway promotes blood-brain barrier integrity and $\mathrm{CNS}$ immune quiescence. Science. 2011;334(6063):1727-1731. doi:10.1126/science.1206936

11. Woodroofe MN, Bellamy AS, Feldmann M, Davison AN, Cuzner ML. Immunocytochemical characterisation of the immune reaction in the central nervous system in multiple sclerosis. Possible role for microglia in lesion growth. J Neurol Sci. 1986;74:135-152.

12. Schiffer D, Mellai M, Bovio E, Annovazzi L. The neuropathological basis to the functional role of microglia/macrophages in gliomas. Neurol Sci. 2017;38(9):1571-1577. doi:10.1007/s10072-017-3002-x

13. Louveau A, Smirnov I, Keyes TJ, et al. Structural and functional features of central nervous system lymphatic vessels. Nature. 2015;523:337-341. doi:10.1038/nature14432

14. Wang Z, Lachmann A, Ma'ayan A. Mining data and metadata from the gene expression omnibus. Biophys Rev. 2019;11:103-110. doi:10.1007/s12551-018-0490-8

15. Jiang P, Liu XS. Big data mining yields novel insights on cancer. Nat Genet. 2015;47:103-104. doi:10.1038/ng.3205

16. Holland EC. Gliomagenesis: genetic alterations and mouse models. Nat Rev Genet. 2001;2:120-129. doi:10.1038/35052535

17. Wang J, Cazzato E, Ladewig E, et al. Clonal evolution of glioblastoma under therapy. Nat Genet. 2016;48:768-776. doi:10.1038/ng.3590

18. Liau BB, Sievers C, Donohue LK, et al. Adaptive chromatin remodeling drives glioblastoma stem cell plasticity and drug tolerance. Cell Stem Cell. 2017;20(2):233-46 e7. doi:10.1016/j.stem.2016.11.003

19. Stupp R, Mason WP, van den Bent MJ, et al. Radiotherapy plus concomitant and adjuvant temozolomide for glioblastoma. N Engl J Med. 2005;352:987-996. doi:10.1056/NEJMoa043330

20. Keime-Guibert F, Chinot O, Taillandier L, et al. Radiotherapy for glioblastoma in the elderly. $N$ Engl J Med. 2007;356(15):1527-1535. doi:10.1056/NEJMoa065901

21. Sturm D, Bender S, Jones DT, et al. Paediatric and adult glioblastoma: multiform (epi)genomic culprits emerge. Nat Rev Cancer. 2014;14(2):92-107. doi:10.1038/nrc3655

22. Kirkwood JM, Butterfield LH, Tarhini AA, Zarour H, Kalinski P, Ferrone S. Immunotherapy of cancer in 2012. CA Cancer J Clin. 2012;62:309-335.

23. Hamilton JD, Rapp M, Schneiderhan T, et al. Glioblastoma multiforme metastasis outside the CNS: three case reports and possible mechanisms of escape. $J$ Clin Oncol. 2014;32(22):e80-4. doi:10.1200/JCO.2013.48.7546

24. Muller C, Holtschmidt J, Auer M, et al. Hematogenous dissemination of glioblastoma multiforme. Sci Transl Med. 2014;6(247):247ra101. doi:10.1126/scitranslmed.3009095

25. Bloch O, Crane CA, Kaur R, Safaee M, Rutkowski MJ, Parsa AT. Gliomas promote immunosuppression through induction of B7-H1 expression in tumor-associated macrophages. Clin Cancer Res. 2013;19:3165-3175. doi:10.1158/1078-0432.CCR-12-3314
26. Hilf N, Kuttruff-Coqui S, Frenzel K, et al. Actively personalized vaccination trial for newly diagnosed glioblastoma. Nature. 2019;565(7738):240-245. doi:10.1038/s41586-018-0810-y

27. Jones SA, Jenkins BJ. Recent insights into targeting the IL-6 cytokine family in inflammatory diseases and cancer. Nat Rev Immunol. 2018;18:773-789. doi:10.1038/s41577-018-0066-7

28. Chan LC, Li CW, Xia W, et al. IL-6/JAK1 pathway drives PD-L1 Y112 phosphorylation to promote cancer immune evasion. J Clin Invest. 2019;129:3324-3338. doi:10.1172/JCI126022

29. Duluc D, Delneste Y, Tan F, et al. Tumor-associated leukemia inhibitory factor and IL-6 skew monocyte differentiation into tumor-associated macrophage-like cells. Blood. 2007;110:4319-4330. doi:10.1182/blood2007-02-072587

30. Lyu X, Zhang M, Li G, Cai Y, Li G, Qiao Q. Interleukin-6 production mediated by the IRE1-XBP1 pathway confers radioresistance in human papillomavirus-negative oropharyngeal carcinoma. Cancer Sci. 2019;110(8):2471-2484. doi:10.1111/cas.14094

31. Tamatani T, Azuma M, Ashida Y, et al. Enhanced radiosensitization and chemosensitization in NF-kappaB-suppressed human oral cancer cells via the inhibition of gamma-irradiation- and 5-FU-induced production of IL-6 and IL-8. Int J Cancer. 2004;108(6):912-921. doi:10.1002/ijc.11640

32. Chen X, Chen F, Ren Y, et al. IL-6 signaling contributes to radioresistance of prostate cancer through key DNA repair-associated molecules ATM, ATR, and BRCA 1/2. J Cancer Res Clin Oncol. 2019;145(6):1471-1484. doi:10.1007/s00432-019-02917-z

33. Sugimura K, Miyata $H$, Tanaka $K$, et al. Let-7 expression is a significant determinant of response to chemotherapy through the regulation of IL-6/STAT3 pathway in esophageal squamous cell carcinoma. Clin Cancer Res. 2012;18:5144-5153. doi:10.1158/ 1078-0432.CCR-12-0701

34. Garbers C, Heink S, Korn T, Rose-John S. Interleukin-6: designing specific therapeutics for a complex cytokine. Nat Rev Drug Discov. 2018;17:395-412. doi:10.1038/nrd.2018.45

35. DePaolo RW, Kamdar K, Khakpour S, Sugiura Y, Wang W, Jabri B. A specific role for TLR1 in protective $\mathrm{T}(\mathrm{H}) 17$ immunity during mucosal infection. J Exp Med. 2012;209(8):1437-1444. doi:10.1084/jem.20112339

36. Ou DL, Chen CL, Lin SB, Hsu CH, Lin LI. Chemokine receptor expression profiles in nasopharyngeal carcinoma and their association with metastasis and radiotherapy. J Pathol. 2006;210(3):363-373. doi:10.1002/path.2053

37. Mondini M, Loyher PL, Hamon P, et al. CCR2-dependent recruitment of tregs and monocytes following radiotherapy is associated with TNFalpha-mediated resistance. Cancer Immunol Res. 2019;7:376-387.

38. Kawada K, Taketo MM. Significance and mechanism of lymph node metastasis in cancer progression. Cancer Res. 2011;71(4):1214-1218. doi:10.1158/0008-5472.CAN-10-3277

39. Canadas I, Thummalapalli R, Kim JW, et al. Tumor innate immunity primed by specific interferon-stimulated endogenous retroviruses. Nat Med. 2018;24(8):1143-1150. doi:10.1038/s41591-018-0116-5

40. Wang G, Fu XL, Wang JJ, Guan R, Tang XJ. Novel strategies to discover effective drug targets in metabolic and immune therapy for glioblastoma. Curr Cancer Drug Targets. 2017;17:17-39. doi:10.2174/1568009616666160512145436
OncoTargets and Therapy

\section{Publish your work in this journal}

OncoTargets and Therapy is an international, peer-reviewed, open access journal focusing on the pathological basis of all cancers, potential targets for therapy and treatment protocols employed to improve the management of cancer patients. The journal also focuses on the impact of management programs and new therapeutic

Submit your manuscript here: https://www.dovepress.com/oncotargets-and-therapy-journa agents and protocols on patient perspectives such as quality of life, adherence and satisfaction. The manuscript management system is completely online and includes a very quick and fair peer-review system, which is all easy to use. Visit http://www.dovepress.com/ testimonials.php to read real quotes from published authors. 